\title{
Clinical features and disease progression in moyamoya disease patients with Graves disease
}

\author{
Jian-Bin Chen, MD, Ding Lei, MD, Min He, MD, Hong Sun, MD, Yi Liu, MD, Heng Zhang, MD, \\ Chao You, MD, and Liang-Xue Zhou, MD, PhD
}

\begin{abstract}
Department of Neurosurgery, West China Hospital, West China School of Medicine, Sichuan University, Chengdu, People's Republic of China
\end{abstract}

OBJECT The present study aimed to clarify the incidence and clinical features of disease progression in adult moyamoya disease (MMD) patients with Graves disease (GD) for better management of these patients.

METHODS During the past 18 years, 320 adult Chinese patients at West China Hospital were diagnosed with MMD, and 29 were also diagnosed with GD. A total of 170 patients ( 25 with GD; 145 without GD) were included in this study and were followed up. The mean follow-up was $106.4 \pm 48.6$ months (range 6-216 months). The progression of the occlusive lesions in the major intracranial arteries was measured using cerebral angiography and was evaluated according to Suzuki's angiographic staging. Information about cerebrovascular strokes was obtained from the records of patients' recent clinical visits. Both angiographic progression and strokes were analyzed to estimate the incidences of angiographic progression and strokes using Kaplan-Meier analysis. A multivariate logistic regression model was used to test the effects of sex, age at MMD onset, disease type, strokes, and GD on the onset of MMD progression during follow-up.

RESULTS During follow-up, the incidence of disease progression in MMD patients with GD was significantly higher than in patients without GD $(40.0 \%$ vs $20.7 \%$, respectively; $p=0.036)$. The interval between initial diagnosis and disease progression was significantly shorter in MMD patients with GD than in patients without GD $(p=0.041)$. Disease progression occurred in both unilateral MMD and bilateral MMD, but the interval before disease progression in patients with unilateral disease was significantly longer than in patients with bilateral disease $(p=0.021)$. The incidence of strokes in MMD patients with GD was significantly higher than in patients without GD (48\% vs $26.2 \%$, respectively; $p=0.027)$. The KaplanMeier survival curve showed significant differences in the incidence of disease progression $(p=0.038$, log-rank test) and strokes ( $p=0.031$, log-rank test) between MMD patients with GD and those without GD. Multivariate analysis suggested that GD may contribute to disease progression in MMD (OR 5.97, 95\% Cl 1.24-33.76, $p=0.043$ ).

CONCLUSIONS The incidence of disease progression in MMD patients with GD was significantly higher than that in MMD patients without GD, and GD may contribute to disease progression in MMD patients. The incidence of strokes was significantly higher in MMD patients with GD than in patients without GD. Management guidelines for MMD patients with GD should be developed.

http://thejns.org/doi/abs/10.3171/2014.10.JNS141140

KEY WORDS moyamoya disease; Graves disease; disease progression; stroke; vascular disorders

$\mathrm{M}$ OYAMOYA disease (MMD) is a disorder characterized by stenosis or occlusion of the terminal portion of the internal carotid artery and the proximal portion of the anterior and/or middle cerebral arteries and is accompanied by the formation of netlike collateral vessels, the "moyamoya" vessels. ${ }^{19}$ Graves disease (GD) is an autoimmune disorder in which autoantibodies to the thyroid stimulating hormone (TSH) receptor induce continuous stimulation of the thyroid gland without any negative feedback, thereby inducing hyperthyroidism. ${ }^{1}$ Kushima and colleagues first reported concurrent MMD and GD. ${ }^{19}$ Reports of concurrent presentations of these 2 diseases have gradually increased during the past 2 decades. ${ }^{15,22,23,30,31,33}$ However, the exact reasons why these 2 diseases can commonly occur in the same individual remain undetermined, making it difficult to draw definitive conclusions about the relationship between MMD and GD. Some studies have demonstrated the progression of

ABBREVIATIONS DSA = digital subtraction angiography; $\mathrm{TT} 3$ = free triiodothyronine; $\mathrm{fT} 4$ = free thyroxine; $\mathrm{GD}=$ Graves disease; $\mathrm{MMD}=$ moyamoya disease; $\mathrm{MRA}=\mathrm{MR}$ angiography; $\mathrm{T} 3$ = triiodothyronine; $\mathrm{T} 4$ = thyroxine; $\mathrm{TIA}=$ transient ischemic attack; $\mathrm{TSH}=$ thyroid stimulating hormone.

SUBMITTED May 23, 2014. ACCEPTED October 8, 2014.

INCLUDE WHEN CITING Published online April 10, 2015; DOI: 10.3171/2014.10.JNS141140.

DISCLOSURE This study was supported by grants from the Doctoral Fund of the Ministry of Education of China (20080441210) and the Special Foundation of Sichuan Postdoctoral Fund (2009064). 
occlusive lesions in MMD patients. ${ }^{18,29}$ However, no reports have clarified the stages of disease progression in a large population of patients with both MMD and GD. Therefore, the clinical course and disease progression of these patients are unclear. In this study, we aimed to explore the incidence and clinical features of disease progression in adult MMD patients with GD.

\section{Methods}

\section{Patient Selection}

During the past 18 years (from 1996 to 2013), 320 adult Chinese patients ( $>18$ years at initial diagnosis) were diagnosed with MMD at West China Hospital, Sichuan University. All of these individuals were diagnosed with MMD based on the guidelines set by the Research Committee on MMD of the Japanese Ministry of Health and Welfare. ${ }^{4}$ Each participant signed an informed consent form. This study was carried out in accordance with the Code of Ethics of the World Medical Association (Declaration of Helsinki). This study also received approval from the Ethics Committee of West China School of Medicine, Sichuan University. Of these patients, 29 (9.1\%) (Fig. 1) were also diagnosed with GD by endocrinologists based on the criteria of routine thyroid function tests, including thyroxine (T4), triiodothyronine (T3), free T4 (fT4), free T3 (fT3), and TSH. ${ }^{6}$ We further checked the rate of thyroid uptake of iodine and conducted the TSH receptor antibody test for atypical clinical symptoms such as palpitations, fatigue, and bulimia to exclude temporary hyperthyroidism caused by subacute thyroiditis, painless thyroiditis, and iodine hyperthyroidism.

\section{Antithyroid Treatment}

Of the 29 patients with MMD and GD, 28 patients first received antithyroid drugs (methimazole $10 \mathrm{mg}, 3$ times per day, or propylthiouracil $100 \mathrm{mg}, 3$ times per day) under the guidance of an endocrinologist according to antithyroid therapy criteria. In addition, radioactive iodine therapy for GD was administered to 5 of the 28 patients. $^{6}$ Then, surgical treatment was performed according to the patient's condition. After antithyroid therapy, patients' hyperthyroid symptoms were controlled. During a mean follow-up period of $106.4 \pm 48.6$ months, thyroid hormone levels in MMD patients with GD were maintained in the normal range as shown by tests every 3-6 months. The values of TSH, fT3, and fT4 are shown in Table 1.

\section{Surgical Treatment}

Using preoperative cerebral digital subtraction angiography (DSA), MRI, PET, or ${ }^{133} \mathrm{Xe}$ - or $N$-isopropyl-4-[123I] iodoamphetamine SPECT, we quantitatively measured cerebral blood flow and its reactivity to acetazolamide in 320 MMD patients at least 4 weeks after the onset of symptoms. The affected hemisphere was considered to be the target for surgical revascularization when the tests revealed ischemia and/or low cerebral perfusion or impaired reactivity to acetazolamide (Figs. 2 and 3). According to these criteria or patients' requests, surgical revascularization was performed on 18 sides in 28 patients. Three patients underwent surgical bilateral revascularization, and 12 patients underwent surgery on 1 side (i.e., 12 sides did not undergo surgery). Thus, 15 patients underwent surgical procedures, and 13 (26 sides) patients did not. Those patients who received surgery on both sides were excluded from follow-up. We assessed the progression of disease for those patients who did not undergo surgery. Therefore, 25 (38 sides) patients were included in the group with GD during follow-up (Fig. 1). One hundred forty-five patients (210 sides) of the 280 MMD patients without GD who did

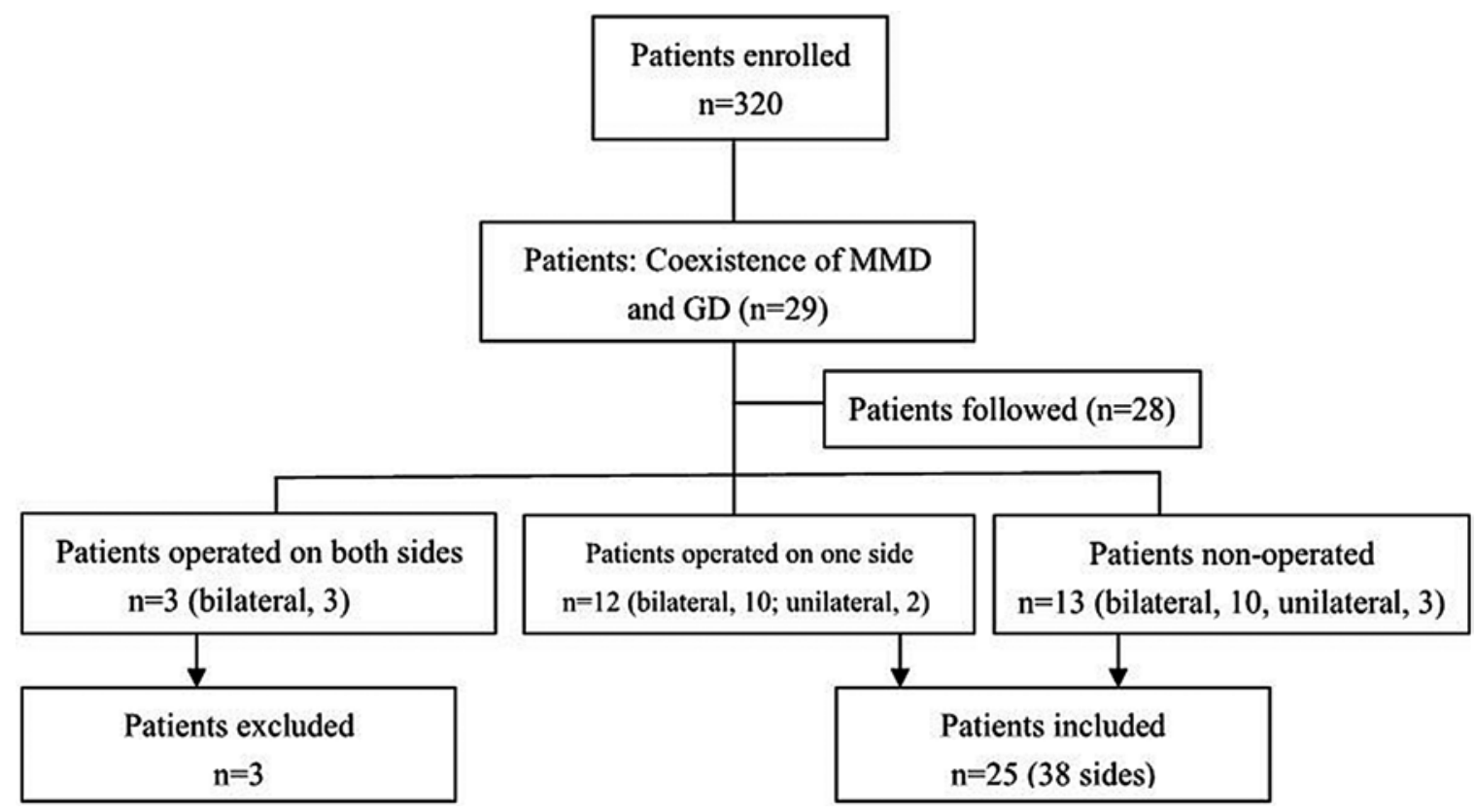

FIG. 1. In this study, 29 (9.1\%) MMD patients were diagnosed with GD, 28 of these patients received treatment, and 25 were included during follow-up. 
not experience surgical revascularization were included during follow-up (Fig. 4). Surgical procedures included superficial temporal artery to middle cerebral artery anastomosis, superficial temporal artery to middle cerebral artery anastomosis combined with encephaloduroarteriosynangiosis, or encephaloduroarteriosynangiosis alone. Therefore, a total of 170 patients ( 25 with GD; 145 without GD) were included in this study during follow-up.

\section{Follow-Up and Evaluation of Disease Progression}

Both MRI and MR angiography (MRA) were performed every 6 or 12 months using a 3.0-T whole-body MR imager. When progression of the occlusive lesion in the major intracranial arteries was suspected, DSA was performed for verification. Occlusive lesions in the carotid branches were graded according to Suzuki's 6 stages of

TABLE 1. Summary of the values of TSH, $f T 3$, and $f T 4$ in MMD patients with $G D^{*}$

\begin{tabular}{|c|c|c|c|c|c|}
\hline \multirow[b]{2}{*}{$\begin{array}{l}\text { Case } \\
\text { No. }\end{array}$} & \multirow[b]{2}{*}{$\begin{array}{l}\text { Age } \\
\text { (yrs) }\end{array}$} & \multirow[b]{2}{*}{ Sex } & \multicolumn{3}{|c|}{$\begin{array}{c}\text { Measurements During } \\
\text { Follow-Up of } 106.4 \pm 48.6 \text { Mos }\end{array}$} \\
\hline & & & $\begin{array}{c}\mathrm{fT} 3 \\
(\mathrm{nmol} / \mathrm{L}) \dagger\end{array}$ & $\begin{array}{c}\mathrm{fT} 4 \\
(\mathrm{pmol} / \mathrm{L}) \dagger\end{array}$ & TSH (mU/L)† \\
\hline 1 & 30 & $\mathrm{~F}$ & $3.7-7.4$ & $17.0-20.5$ & $0.45-1.0$ \\
\hline 2 & 37 & $\mathrm{~F}$ & $4.6-6.9$ & $12.4-16.0$ & $2.7-4.0$ \\
\hline 3 & 41 & $F$ & $5.5-7.5$ & $14.0-18.0$ & $1.78-2.6$ \\
\hline 4 & 44 & $\mathrm{~F}$ & $4.3-7.0$ & $20.0-21.4$ & $3.45-3.80$ \\
\hline 5 & 46 & $\mathrm{~F}$ & $4.0-6.3$ & $13.6-15.0$ & $2.23-2.64$ \\
\hline 6 & 28 & M & $7.0-7.3$ & $15.0-16.8$ & $1.37-2.30$ \\
\hline 7 & 48 & $F$ & $3.8-6.8$ & $12.4-15.5$ & $3.11-4.0$ \\
\hline 8 & 24 & $F$ & $3.61-7.0$ & $18.0-21.4$ & $1.15-2.17$ \\
\hline 9 & 35 & $M$ & $6.5-7.0$ & $16.4-18.5$ & $1.31-3.99$ \\
\hline 10 & 35 & $\mathrm{~F}$ & $4.5-6.3$ & $12.0-13.5$ & $0.482-1.21$ \\
\hline 11 & 38 & $F$ & $3.9-7.1$ & $14.5-16.4$ & $2.17-3.90$ \\
\hline 12 & 32 & $M$ & $4.1-6.5$ & $13.1-22.0$ & $0.45-2.87$ \\
\hline 13 & 25 & $F$ & $4.0-7.2$ & $15.0-18.5$ & $1.21-1.49$ \\
\hline 14 & 29 & $\mathrm{~F}$ & $3.6-5.7$ & $14.0-17.5$ & $2.0-2.35$ \\
\hline 15 & 26 & $\mathrm{~F}$ & $4.4-7.0$ & $12.5-14.3$ & $1.65-2.08$ \\
\hline 16 & 24 & $\mathrm{~F}$ & $3.8-7.1$ & $17.6-20.4$ & $1.49-4.1$ \\
\hline 17 & 26 & $F$ & $3.9-6.5$ & $18.6-20.5$ & $2.82-3.17$ \\
\hline 18 & 51 & $F$ & $3.6-7.0$ & $19.0-21.6$ & $2.46-3.14$ \\
\hline 19 & 39 & $F$ & $4.8-5.6$ & $14.6-18.6$ & $2.73-3.08$ \\
\hline 20 & 24 & $\mathrm{~F}$ & $6.0-6.8$ & $15.5-20.6$ & $2.14-2.61$ \\
\hline 21 & 45 & M & $3.8-7.2$ & $17.4-19.6$ & $0.456-2.57$ \\
\hline 22 & 21 & $\mathrm{~F}$ & $3.8-5.5$ & $20.0-21.5$ & $0.794-1.13$ \\
\hline 23 & 38 & $M$ & $5.3-6.0$ & $12.5-16.6$ & $0.336-0.456$ \\
\hline 24 & 18 & $F$ & $6.6-7.2$ & $18.4-22.0$ & $2.34-3.14$ \\
\hline 25 & 65 & $M$ & $3.9-4.5$ & $13.6-17.4$ & $2.57-3.06$ \\
\hline
\end{tabular}

* The thyroid hormones were examined at 6 -month intervals. The data are shown as the lowest to the highest values of all tests per patient. The values remained in the normal range during follow-up.

† Normal reference values: fT3 $=3.60-7.50 \mathrm{nmol} / \mathrm{L} ; \mathrm{fT} 4=12.0-22.0 \mathrm{pmol} / \mathrm{L}$; and $\mathrm{TSH}=0.27-4.2 \mathrm{mU} / \mathrm{L}$. angiographic staging ${ }^{4,9}$ : Stage I, narrowing of the carotid siphon; Stage II, initiation of moyamoya vessels; Stage III, intensification of moyamoya vessels; Stage IV, reduction of moyamoya vessels; Stage V, minimization of moyamoya vessels; and Stage VI, disappearance of moyamoya vessels. The progression of lesions from lower to higher stages in the carotid branches was graded according to Suzuki's angiographic staging by 1 neurosurgical radiologist and 2 neurosurgeons who were blinded to the patient's GD status. Data about cerebrovascular strokes (including ischemic stroke, transient ischemic attack [TIA], or hemorrhage) were obtained from the records of patients' most recent clinical visits. The mean follow-up was $106.4 \pm$ 48.6 months (range 6-216 months).

\section{Statistical Analysis}

To clarify the predictors of disease progression in MMD with GD, we performed primary comparisons between patients with GD and without GD. Categorical variables were analyzed using a Pearson chi-square test or Fisher exact test. Continuous variables were compared using an independent Student t-test. Continuous variables were expressed as a percentage or as mean \pm SD. Differences were considered to be statistically significant if the $\mathrm{p}$ value was $<0.05$. The incidences of both angiographic progression and strokes were analyzed using KaplanMeier analysis. The log-rank test was used to analyze the survival differences between patients with GD and without GD. Subsequently, a multivariate logistic regression analysis was conducted to test the effects of sex, age at MMD onset, disease type, cerebrovascular strokes, and GD on the onset of MMD progression during follow-up. The statistical level of significance was also set at $\mathrm{p}<0.05$. Statistical analysis was completed with SPSS 13.0.

\section{Results}

\section{Clinical Characteristics}

Of the 320 patients, 148 were male and 172 were female. The average age was $36.1 \pm 12.9$ years (range $18-68$ years) at MMD onset. The prevalence of GD in MMD patients was $9.1 \%(n=29)$ (Fig. 1). The ratio of males to females in the 29 MMD patients with GD (male/female 1:

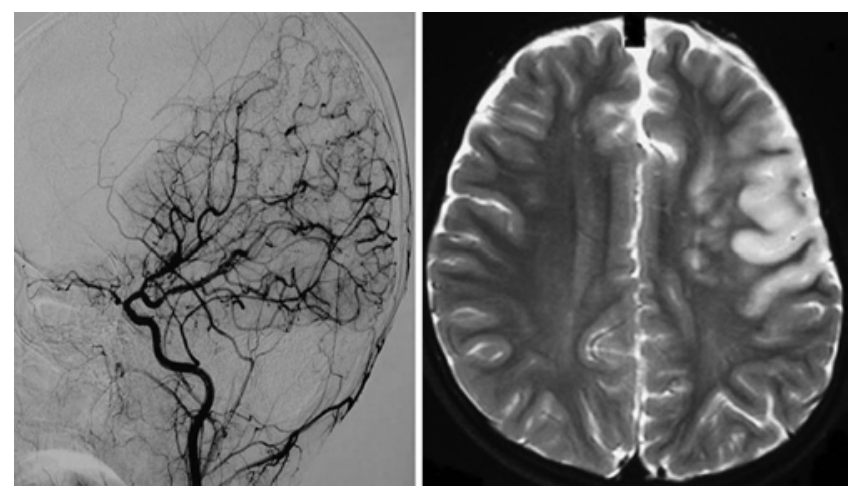

FIG. 2. Left: Cerebral DSA image revealing stenosis and occlusion of the left internal carotid artery bifurcation. Right: Magnetic resonance image showing ischemic lesions in the left frontal and parietal lobes. 


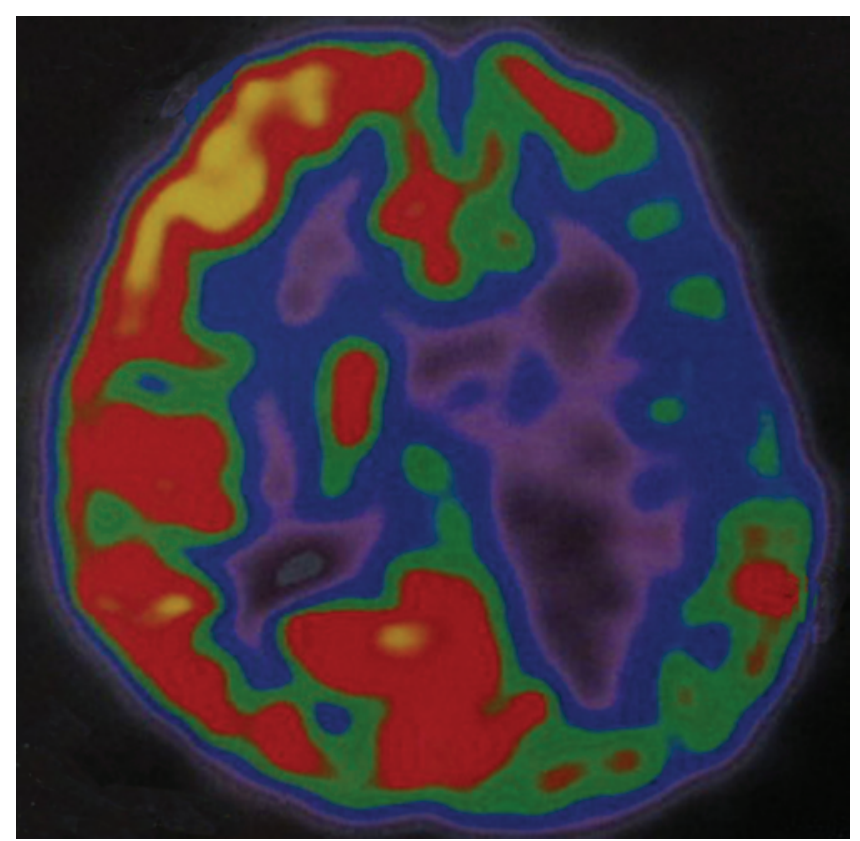

FIG. 3. PET scan revealing low cerebral perfusion and glucose metabolism and indicating ischemia in the left frontal and parietal lobes. Figure is available in color online only.

3.1) was significantly higher than that in the whole sample $(\mathrm{p}=0.030)$, and the mean age was $34.8 \pm 11.1$ years (range 18-65 years) at MMD onset. Of the 28 patients, the initial symptoms were TIA, cerebral infarction, and hemorrhage in 5, 14, and 9 patients, respectively. Twenty-three patients had bilateral involvement, and 5 had unilateral moyamoya vessels.

\section{Features of Disease Progression in MMD Patients With GD}

During the follow-up period, the occlusive lesions in the major intracranial arteries progressed in 10 (26.3\%) of 38 nonoperated sides or in $10(40.0 \%)$ of 25 patients. Disease progression was verified in 1 man and 9 women, whose age at disease onset was $34.4 \pm 9.5$ years (range 23-47 years). The interval between initial MMD diagnosis and disease progression was significantly shorter in patients with GD (mean $40.2 \pm 7.5$ months, range 2 months to 5 years) than in patients without GD (mean $59.6 \pm 17.4$ months, range 8 months to 5 years) $(\mathrm{p}=0.041)$. Disease progression occurred in all 5 patients $(100 \%)$ with unilateral MMD, and the interval between initial diagnosis and disease progression was $40.6 \pm 22.4$ months (range 10 months to 5.0 years). Five (25.0\%) of 20 patients with bilateral MMD showed progression, and the interval between initial diagnosis and disease progression varied from 7 months to 5.6 years (mean $45.6 \pm 26.7$ months). The incidence of disease progression in patients with GD $(40.0 \%)$ was significantly higher than in patients without GD $(20.7 \%)(\mathrm{p}=0.036)$. Of all of these 25 patients with GD who were followed up, the strokes included intracranial bleeding in 3 patients, TIA in 1, and ischemia in 8 . A PET study revealed the deterioration of cerebral hemodynamics in 5 patients. Four patients experienced as many as 5 strokes per patient during follow-up. The incidence of stroke in MMD patients with GD (12 [48\%] of 25) was significantly higher than that in MMD patients without GD $(38$ [26.2\%] of 145) $(p=0.027)$. Seven of 25 patients with

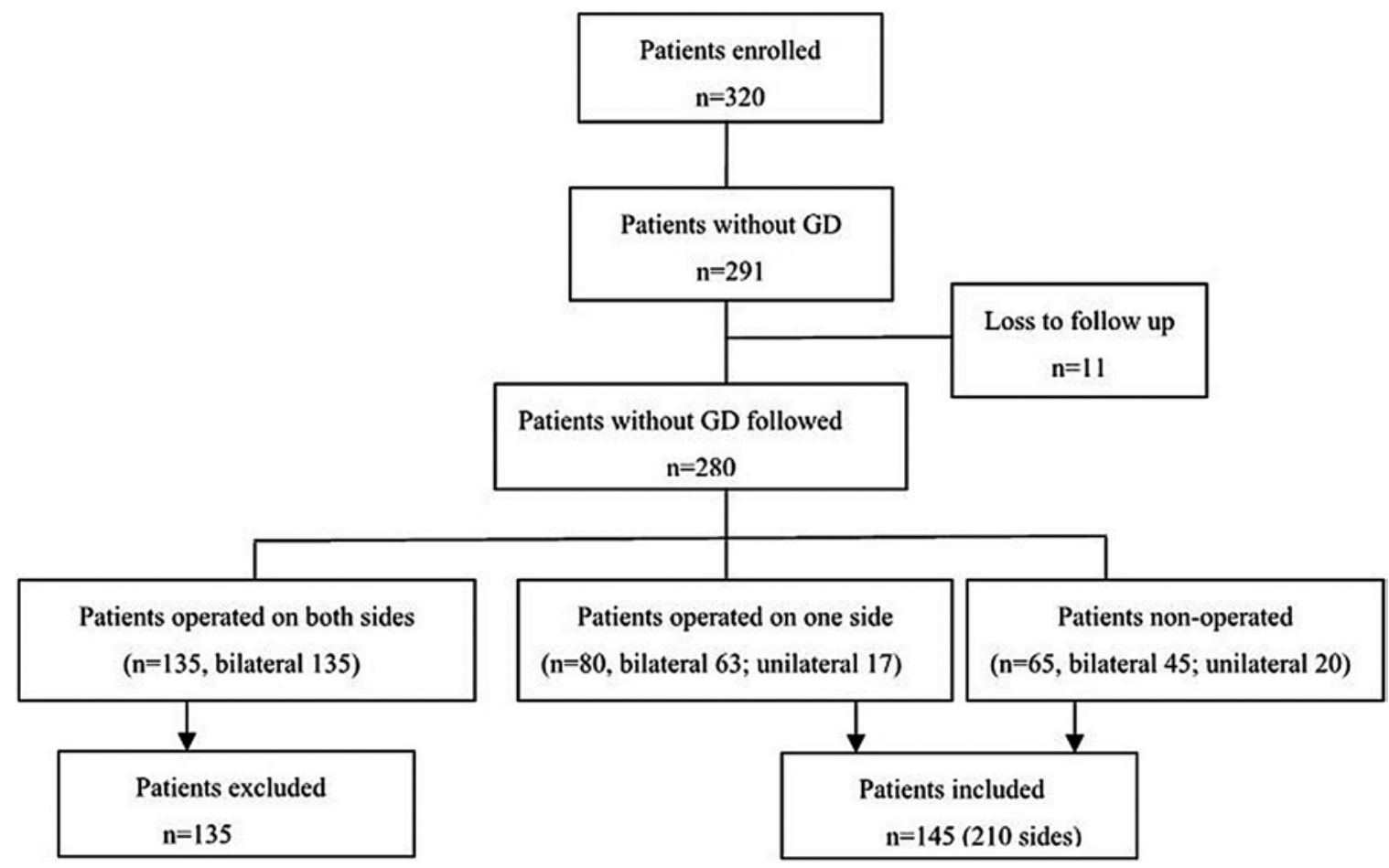

FIG. 4. Overall, 145 MMD patients without GD were included during follow-up. 
GD underwent additional bypass surgery, and 12 required medical treatment. One patient died following a third occurrence of intracranial bleeding in the 7th year following the onset of symptoms.

\section{Features of Disease Progression in MMD Patients Without GD}

In our study, 145 patients (210 nonoperated sides) without GD were observed during follow-up (Fig. 4). The occlusive lesions in the major intracranial arteries progressed in $30(14.3 \%)$ of 210 sides or in 30 (20.7\%) of 145 patients; of these 30 patients, 23 were women and 7 were men. The age at disease onset was $36.4 \pm 12.6$ years (range 18-71 years), and the interval between initial diagnosis and disease progression was $59.6 \pm 17.4$ months (range 8 months to 5 years). During follow-up, intracranial bleeding occurred in 17, TIA in 8 , and ischemia in 13 of 145 patients. A PET study revealed the deterioration of cerebral hemodynamics in 5 patients. On the one hand, disease progression occurred in $15(40.5 \%)$ of 37 patients with unilateral MMD, and the interval between initial diagnosis and disease progression varied from 1.3 to 9 years (mean $65.2 \pm 32.7$ months). On the other hand, disease progression occurred in 15 (13.9\%) of 108 patients with bilateral MMD, and the interval between initial diagnosis and disease progression varied from 1.0 to 13 years (mean $64.6 \pm 33.8$ months). Fifteen of 108 patients with bilateral MMD showed progression of the occlusive lesion in the carotid branches, and 4 patients with bilateral MMD developed an additional occlusive lesion in the posterior cerebral artery during follow-up. The interval between diagnosis and disease progression in patients with unilateral MMD was significantly longer than that in patients with bilateral MMD $(\mathrm{p}=0.021)$. During the follow-up period, intracranial bleeding occurred in 10, TIA in 6 , and ischemia in 14 of 30 patients whose disease progressed, and a PET study revealed deterioration of cerebral hemodynamics in 9 patients. Surgery was performed to evacuate hemorrhages in 10 of 30 patients with cerebral bleeding, and 9 patients with deterioration of cerebral he- modynamics underwent additional bypass surgery. All of these 30 patients received standard medical treatment.

\section{Kaplan-Meier Survival Estimates}

The Kaplan-Meier survival curve showed that the difference in angiographic progression between MMD patients with GD and those without GD reached statistical significance $(\mathrm{p}=0.038$, log-rank test [Fig. 5 left]) and that the incidence of angiographic progression was significantly higher in MMD patients with GD than in patients without GD, as shown in Fig. 5. The Kaplan-Meier curve also showed that the difference in strokes between the 2 groups reached statistical significance $(p=0.031, \log$-rank test [Fig. 5 right]), and the incidence of strokes was significantly higher in MMD patients with GD than in patients without GD, as shown in Fig. 5.

\section{Independent Predictor of Disease Progression}

The effects of various clinical factors on disease progression in MMD patients with GD and without GD were compared. After univariate analysis, no significant correlations were observed with respect to patient age, history of stroke, and disease type between the 2 groups. However, disease progression was noted in $9(47.4 \%)$ of 19 female patients with GD and in 23 (28.8\%) of 80 female patients without GD, revealing that the incidence of disease progression among female patients was not significantly different (Pearson chi-square test, $\mathrm{p}=0.119$ ) between patients with GD and without GD. After adjustments for covariates such as sex, age at disease onset, disease type, GD, and cerebrovascular strokes, the multivariate analysis suggested that GD may contribute to disease progression in MMD (OR 5.97, 95\% CI 1.24-33.76, $\mathrm{p}=0.043$ ).

\section{Discussion}

The natural history of MMD has previously been outlined in a number of studies. ${ }^{16,17}$ The coexistence of MMD and GD has been increasingly reported in recent years.
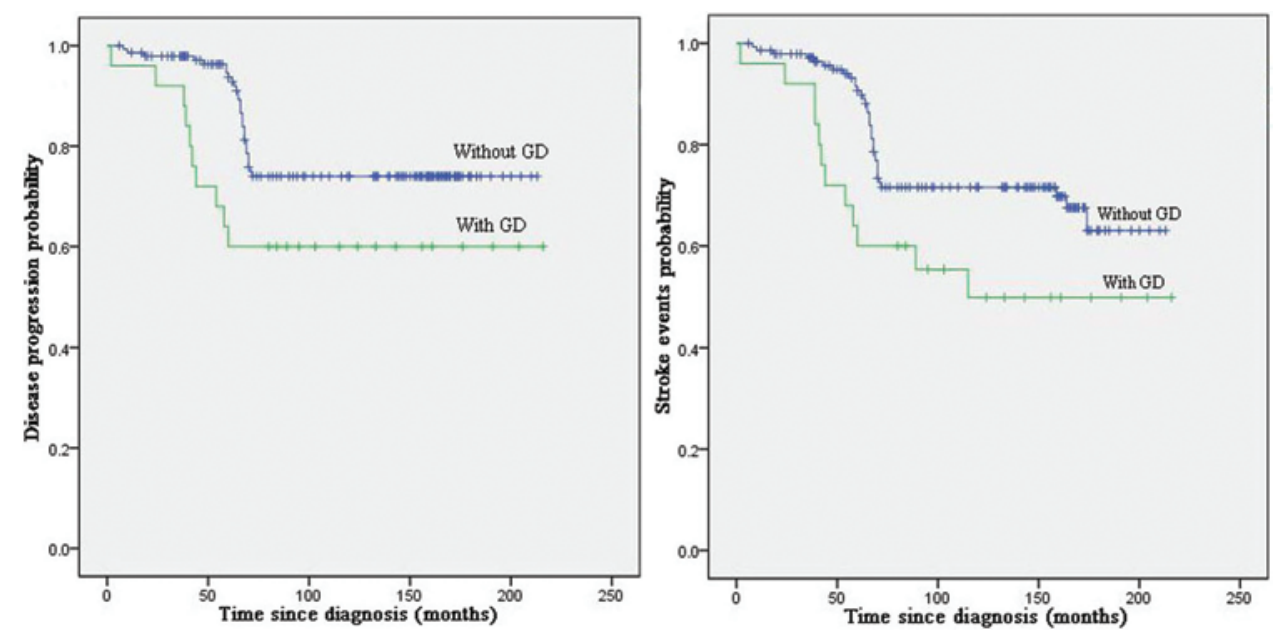

FIG. 5. Kaplan-Meier survival curves showing that the differences in angiographically determined disease progression $(p=0.038$, log-rank test [left]) and strokes ( $p=0.031$, log-rank test [right]) between patients with GD and without GD, respectively, reached statistical significance. Figure is available in color online only. 
This study is the first to focus on progression and clinical manifestations in the major intracranial arteries in a large population of patients with concurrent MMD and GD. Although thyroid hormone levels were controlled within the normal range in patients with GD, the results clearly showed that the incidence of disease progression was higher in these patients than in patients without GD and also that it was higher than previously recognized. ${ }^{18}$ Multivariate analysis showed that MMD patients with GD had a higher risk of disease progression than did MMD patients without GD. The interval between initial diagnosis and disease progression was significantly shorter in patients with GD than in patients without GD. A significant association between a history of GD and recurrent stroke was observed. The incidence of strokes in patients with GD was significantly higher than in patients without GD. This study indicated that GD may be associated with disease progression and cerebrovascular consequences in MMD patients.

Disease progression in adult MMD patients previously was considered very rare. Kuroda et al. reported that disease progression occurred in 15 of 63 adult patients, including 4 patients with unilateral MMD and 11 patients with bilateral MMD; until now, theirs was the largest population in which the progression of MMD was studied. ${ }^{18}$ Several studies have also reported the progression of occlusive lesions in adult MMD patients. ${ }^{8-10}$ In addition, several studies have reported that unilateral MMD has progressed to the bilateral type., ${ }^{3,11,13,34}$ Thus, disease progression can occur in adult MMD patients. To our knowledge, more than 78 concurrent cases of GD and MMD or moyamoya syndrome have been previously described. Moreover, 2 studies described the association between MMD and thyroid autoantibodies and/or thyroid dysfunction..$^{15,23}$ However, fewer than 10 adult MMD patients with GD have been reported to exhibit progression according to case reports. Ezura et al. examined the course of changes in 6 cases of MMD using cerebral angiography and reported that disease progression was found in 11 hemispheres of 12 patients between childhood (15 years and younger) and adolescence (15-20 years), whereas progression was found in only 3 hemispheres of 12 patients between adolescence and adulthood. ${ }^{2}$ Therefore, they concluded that MMD progressed between childhood and adolescence and stopped or progressed little from adolescence to adulthood. In the present study, however, the results indicated that disease continued to progress in adult MMD patients. In particular, the incidence of disease progression was higher in MMD patients with GD than in patients without GD, and the interval from initial diagnosis to disease progression was shorter in patients with GD than in patients without GD. The findings suggest that disease progression in adult MMD with GD was active.

This raises the question of why MMD patients with GD tend to experience disease progression. We analyzed data obtained in MMD patients with GD in whom disease progression in moyamoya-affected vessels had previously been reported. ${ }^{8,19,22,30}$ Based on several earlier reports, we know that almost all MMD patients develop symptoms during the hyperthyroid state. During the thyrotoxic state, excessive production of thyroid hormones is believed to increase cerebral metabolism and oxygen consumption, resulting in impaired cerebral perfusion. Thus, the cerebrovascular hemodynamics of MMD patients with thyrotoxicosis can result in cerebrovascular events and may cause alterations of the arterial walls. In addition, some MMD patients experienced stroke soon after they received antithyroid treatment or shortly after their antithyroid drug dosage was increased ${ }^{20,24}$ suggesting that hemodynamic changes elicited by a sudden surge of thyroid hormone may exacerbate vascular disease in MMD patients. Moreover, some individuals repeatedly experienced additional cerebral infarction following relapse to hyperthyroidism..$^{8,12,25,32}$ Some studies have also suggested that abnormal thyroid function-especially fluctuations of thyroid function caused by hyperthyroidism-may be an important risk factor that can trigger or aggravate symptoms or disease progression in MMD patients. ${ }^{14,27,28}$ Thyroid hormones may augment vascular sensitivity to the sympathetic nervous system and may induce pathological changes in the arterial walls. An immune-mediated mechanism may play a role in the pathogenesis of these diseases. ${ }^{21}$ Vasculitis induced by antithyroid drugs may cause changes in the intracranial arteries. Thyrotoxic states may accelerate the progressive occlusive disease in the intracranial arteries. ${ }^{7,26}$ High levels of thyroid hormone in GD patients damaged their arterial walls by altering vascular reactivity, and GD has been hypothesized to lead to moyamoya syndrome. ${ }^{5,24,32}$ Lee et al. reported that strict control of thyroid function in patients with hyperthyroidism may help prevent the associated vascular abnormalities..$^{20}$ According to multivariate analysis in the study reported here, GD may be an independent factor for disease progression in adult MMD patients, suggesting an underlying common pathogenic mechanism between these 2 diseases. The antithyroid treatment did not significantly change disease progression in MMD, and the fluctuations of thyroid hormone levels may be an important factor. In addition, the immune-mediated repeated stimulation of the walls of arteries may play an important role in disease progression.

In the present study, disease progression occurred more often in females than in males. However, in this study, the association of sex and disease progression was not significantly different between MMD patients with GD and those without GD. The sex difference may be attributed to GD, which mainly affects females. ${ }^{6}$ Kuroda et al. reported female sex may be a significant predictor of disease progression in adult MMD patients. ${ }^{18}$ Furthermore, female predominance is more pronounced in familial MMD. A study also showed that male/female ratios were 1:1.5 and 1:1.6 in familial and sporadic cases, respectively, indicating female predominance in familial MMD..$^{15}$ Although the sex difference in disease progression between patients with and without GD was not significant, the incidence in female patients was higher than that in male patients in this study.

This study revealed that $40.0 \%$ of patients with GD experienced disease progression of moyamoya vessels. The incidence of disease progression in patients with GD was much higher than that in patients without GD and was larger than previously believed. ${ }^{18}$ The interval between initial diagnosis and disease progression was significantly 
shorter in patients with GD than in patients without GD. Mean follow-up periods were 3 to 6 years in previous studies. The patients included in the present study were followed up for a mean period of 106.4 months (approximately 8.9 years). In addition, all 5 patients with unilateraltype disease and GD progressed to typical bilateral-type MMD. However, 15 of 37 patients without GD showed progression from unilateral- to bilateral-type MMD. The interval for unilateral-type progression to bilateral-type MMD was shorter in patients with GD than in patients without GD. The findings suggested that MMD in patients with GD could easily progress, even if the levels of thyroid hormones were controlled within the normal range during follow-up.

During follow-up, strokes occurred in 12 of 25 patients with GD. Recurrent strokes occurred in 4 patients, and the maximum recurrence was up to 5 times during follow-up of 10 years. However, 38 of 145 patients without GD experienced strokes during follow-up, and no recurrent strokes were observed. The incidence of strokes was significantly higher in MMD patients with GD than in patients without GD. The results indicated that both patients with GD and without GD should be carefully observed and followed up for better management.

\section{Conclusions}

This study suggests that the incidence of disease progression in adult MMD with GD is significantly higher than in adult MMD without GD, and GD may contribute to disease progression. Disease progression in MMD patients with GD is very active. Disease progression can occur in patients with unilateral MMD with GD and in those with bilateral MMD with GD. In addition, the incidence of strokes is much higher in patients with GD than in patients without GD. Management guidelines for MMD patients with GD should be established.

\section{References}

1. Armengol MP, Sabater L, Fernández M, Ruíz M, Alonso N, Otero MJ, et al: Influx of recent thymic emigrants into autoimmune thyroid disease glands in humans. Clin Exp Immunol 153:338-350, 2008

2. Ezura M, Yoshimoto T, Fujiwara S, Takahashi A, Shirane R, Mizoi K: Clinical and angiographic follow-up of childhoodonset moyamoya disease. Childs Nerv Syst 11:591-594, 1995

3. Fujiwara F, Yamada H, Hayashi S, Tamaki N: [A case of adult moyamoya disease showing fulminant clinical course associated with progression from unilateral to bilateral involvement.] No Shinkei Geka 25:79-84, 1997 (Jpn)

4. Fukui M: Guidelines for the diagnosis and treatment of spontaneous occlusion of the circle of Willis ('moyamoya' disease). Research Committee on Spontaneous Occlusion of the Circle of Willis (Moyamoya Disease) of the Ministry of Health and Welfare, Japan. Clin Neurol Neurosurg 99 Suppl 2:S238-S240, 1997

5. Garcin B, Louissaint T, Hosseini H, Blanc R, Fénelon G: Reversible chorea in association with Graves' disease and moyamoya syndrome. Mov Disord 23:620-622, 2008

6. Ginsberg J: Diagnosis and management of Graves' disease. CMAJ 168:575-585, 2003

7. Golomb MR, Biller J, Smith JL, Edwards-Brown M, Sanchez
JC, Nebesio TD, et al: A 10-year-old girl with coexistent moyamoya disease and Graves' disease. J Child Neurol 20:620-624, 2005

8. Hsu SW, Chaloupka JC, Fattal D: Rapidly progressive fatal bihemispheric infarction secondary to Moyamoya syndrome in association with Graves thyrotoxicosis. AJNR Am J Neuroradiol 27:643-647, 2006

9. Im SH, Oh CW, Kwon OK, Kim JE, Han DH: Moyamoya disease associated with Graves disease: special considerations regarding clinical significance and management. J Neurosurg 102:1013-1017, 2005

10. Inaba M, Henmi Y, Kumeda Y, Ueda M, Nagata M, Emoto $\mathrm{M}$, et al: Increased stiffness in common carotid artery in hyperthyroid Graves' disease patients. Biomed Pharmacother 56:241-246, 2002

11. Kagawa R, Okada Y, Moritake K, Takamura M: Magnetic resonance angiography demonstrating adult moyamoya disease progressing from unilateral to bilateral involvementcase report. Neurol Med Chir (Tokyo) 44:183-186, 2004

12. Kamasaki H, Takeuchi T, Mikami T, Komeichi K, Tsutsumi $\mathrm{H}$ : A case of graves' disease diagnosed in the course of bilateral carotid artery stenoses (moyamoya disease); a case report and review of the literature. Clin Pediatr Endocrinol 22:39-44, 2013

13. Kawano T, Fukui M, Hashimoto N, Yonekawa Y: Follow-up study of patients with "unilateral" moyamoya disease. Neurol Med Chir (Tokyo) 34:744-747, 1994

14. Kelly ME, Bell-Stephens TE, Marks MP, Do HM, Steinberg GK: Progression of unilateral moyamoya disease: a clinical series. Cerebrovasc Dis 22:109-115, 2006

15. Kim SJ, Heo KG, Shin HY, Bang OY, Kim GM, Chung CS, et al: Association of thyroid autoantibodies with moyamoyatype cerebrovascular disease: a prospective study. Stroke 41:173-176, 2010

16. Kuriyama S, Kusaka Y, Fujimura M, Wakai K, Tamakoshi A, Hashimoto S, et al: Prevalence and clinicoepidemiological features of moyamoya disease in Japan: findings from a nationwide epidemiological survey. Stroke 39:42-47, 2008

17. Kuroda S, Houkin K: Moyamoya disease: current concepts and future perspectives. Lancet Neurol 7:1056-1066, 2008

18. Kuroda S, Ishikawa T, Houkin K, Nanba R, Hokari M, Iwasaki Y: Incidence and clinical features of disease progression in adult moyamoya disease. Stroke 36:2148-2153, 2005

19. Kushima K, Satoh Y, Ban Y, Taniyama M, Ito K, Sugita K: Graves' thyrotoxicosis and Moyamoya disease. Can J Neurol Sci 18:140-142, 1991

20. Lee R, Sung K, Park YM, Yu JJ, Koh YC, Chung S: A case of Moyamoya disease in a girl with thyrotoxicosis. Yonsei Med J 50:594-598, 2009

21. Leno C, Mateo I, Cid C, Berciano J, Sedano C: Autoimmunity in Down's syndrome: another possible mechanism of Moyamoya disease. Stroke 29:868-869, 1998

22. Li D, Yang W, Xian P, Liu P, Bao X, Zong R, et al: Coexistence of moyamoya and Graves' diseases: the clinical characteristics and treatment effects of 21 Chinese patients. Clin Neurol Neurosurg 115:1647-1652, 2013

23. Li H, Zhang ZS, Dong ZN, Ma MJ, Yang WZ, Han C, et al: Increased thyroid function and elevated thyroid autoantibodies in pediatric patients with moyamoya disease: a case-control study. Stroke 42:1138-1139, 2011

24. Liu JS, Juo SH, Chen WH, Chang YY, Chen SS: A case of Graves' diseases associated with intracranial moyamoya vessels and tubular stenosis of extracranial internal carotid arteries. J Formos Med Assoc 93:806-809, 1994

25. Nakamura K, Yanaka K, Ihara S, Nose T: Multiple intracranial arterial stenoses around the circle of Willis in association with Graves' disease: report of two cases. Neurosurgery 53:1210-1215, 2003

26. Ni J, Gao S, Cui LY, Li SW: Intracranial arterial occlusive 
lesion in patients with Graves' disease. Chin Med Sci J 21:140-144, 2006

27. Piao R, Oku N, Kitagawa K, Imaizumi M, Matsushita K, Yoshikawa T, et al: Cerebral hemodynamics and metabolism in adult moyamoya disease: comparison of angiographic collateral circulation. Ann Nucl Med 18:115-121, 2004

28. Sasaki T, Nogawa S, Amano T: Co-morbidity of moyamoya disease with Graves' disease. Report of three cases and a review of the literature. Intern Med 45:649-653, 2006

29. Shirane R, Mikawa S, Ebina T: A case of adult moyamoya disease showing progressive angiopathy on cerebral angiography. Clin Neurol Neurosurg 101:210-214, 1999

30. Suzuki S, Mitsuyama T, Horiba A, Fukushima S, Hashimoto N, Kawamata T: Moyamoya disease complicated by Graves' disease and type 2 diabetes mellitus: report of two cases. Clin Neurol Neurosurg 113:325-329, 2011

31. Tendler BE, Shoukri K, Malchoff C, MacGillivray D, Duckrow R, Talmadge T, et al: Concurrence of Graves' disease and dysplastic cerebral blood vessels of the moyamoya variety. Thyroid 7:625-629, 1997

32. Tsai MH, Tan TY, Kuo YL, Chang KC: Multiple intracranial arterial stenoses in association with thyrotoxicosis: a case report. Acta Neurol Taiwan 15:105-108, 2006

33. Utku U, Asil T, Celik Y, Tucer D: Reversible MR angio- graphic findings in a patient with autoimmune Graves disease. AJNR Am J Neuroradiol 25:1541-1543, 2004

34. Wanifuchi H, Takeshita M, Aoki N, Kawamata T, Shiokawa $\mathrm{K}$, Izawa M, et al: Adult moyamoya disease progressing from unilateral to bilateral involvement. Neurol Med Chir (Tokyo) 36:87-90, 1996

\section{Author Contributions}

Conception and design: all authors. Acquisition of data: all authors. Analysis and interpretation of data: all authors. Drafting the article: Zhou, Chen, Lei. Critically revising the article: Zhou, Chen, Zhang, You. Reviewed submitted version of manuscript: Zhou, Chen. Approved the final version of the manuscript on behalf of all authors: Zhou. Statistical analysis: Zhou, Chen. Administrative/technical/material support: Zhou, Chen. Study supervision: Zhou, Chen.

\section{Correspondence}

Liang-Xue Zhou, Department of Neurosurgery, West China Hospital, West China School of Medicine, Sichuan University, No. 37, Guoxue Alley, Chengdu 610041, Sichuan Province, People's Republic of China. email: 971672020@qq.com. 